\title{
Entropy estimation with suffix arrays
}

\author{
Kunihiko SADAKANE ${ }^{1}$ \\ ${ }^{1}$ National Institute of Informatics
}

\begin{abstract}
In this paper we give an algorithm for estimating the entropy of a string using the suffix array. Our algorithm is based on a new combinatorial property of the longest common prefix array of a string.
\end{abstract}

\section{KEYWORDS}

Strings, entropy estimation, suffix arrays

\section{Introduction}

This paper proposes a fast algorithm for entropy estimation of a string. There exists an entropy estimation scheme based on the LZ77 compression algorithm (See Section 2.2 for details). However, the LZ77 compression algorithm requires dynamic data structures which are in practice slow. The algorithm proposed in this paper uses a static data structure called a suffix array which can be constructed quickly, and returns the same estimation value with that using LZ77. Our data structure can also compute another estimation value of the entropy (See Section 2.1).

\section{Known algorithms for entropy esti- mation}

In this section we review two known algorithms for entropy estimation for strings. Throughout this paper, we use the following definitions and notations. For a string $S=S[0] S[1] \ldots$, let $S_{j}$ denote $S[j] S[j+1] \ldots$ and it is called a suffix of $S$. Let $\operatorname{lcp}(\alpha, \beta)$ denote the length of the longest common prefix of two strings $\alpha$ and $\beta$.

\subsection{Using distinguishing prefixes}

Definition 1 For a string $S$, we define distinguishing prefix of a suffix $S_{j}$ as the shortest prefix of $S_{j}$ that does not match with prefixes of any other suffixes. We also define $B_{j}$ as the length of the distinguishing prefix of the suffix $S_{j}$.

Received November 1, 2011; Accepted December 22, 2011.

1) sada@nii.ac.jp,http://researchmap.jp/sada/

DOI: 10.2201/NiiPi.2012.9.4
Example: Let $S[0 . .13]=$ "tobeornottobe $\$$ ". The distinguishing prefix of a suffix $S_{8}=$ "ttobe $\$$ " is " $t$ " and therefore $B_{8}=2$.

Yokoo [1], [2] proposed an algorithm to incrementally estimate the entropy of a string. He uses the average value of $B_{j}$ to estimate the entropy. He also proposed an expected linear time algorithm to maintain a list of prefixes which are in lexicographic order of reversed strings. Though it seems to be natural to estimate the entropy by $B_{j}$, Yokoo showed that the entropy estimated by the average value of $B_{j}$ is sometimes far from real compression ratio of many compression algorithms. Therefore we use another entropy estimator.

\subsection{Using the LZ77 algorithm}

Definition 2 We define $L_{n}(j)$ as the smallest integer $L(>0)$ which satisfies $x_{j}^{j+L-1} \neq x_{i}^{i+L-1}, j-n \leq \forall i<j$.

$L_{n}(j)$ stands for the length of a prefix of a suffix $S_{j}$ such that the prefix does not match with prefixes of suffixes $S_{j-n}$ to $S_{j-1}$. For a random string $X$, let $L_{n}(X)$ denote a random variable for $L_{n}(0)$.

Example: Let $S[0 . .13]=$ "tobeornottobe\$". Since $S_{9}=$ "to be $\$$ " matches with the prefix of $S_{0}$ by 4 characters, $L_{9}(9)=5$. On the other hand, $S_{9}$ matches with a prefix of suffixes $S_{1}, \ldots, S_{8}$ by 1 character, and therefore $L_{8}(9)=2$.

The difference between $B_{j}$ and $L_{n}(j)$ is that $L_{n}(j)$ is defined as the length of prefix of $S_{j}$ which does not appear in prefixes of suffixes preceding $S_{j}$, while $B_{j}$ is defined as distinguishing length between $S_{j}$ and both 
preceding and following suffixes. The value $L_{n}(j)$ corresponds to the length of a prefix of $S_{j}$ encoded by the LZ77 algorithm [3]. The following theorem proves asymptotical optimality of the LZ77 code.

Theorem 1 (Wyner, Ziv [4]) As $n \rightarrow \infty$,

$$
\frac{\log n}{L_{n}(X)} \rightarrow H, \text { in probability, }
$$

where $H$ is the entropy of a stationary ergodic information source.

We use the average value of $L_{j}(j)$

$$
\sum_{j=0}^{n-1} L_{j}(j)
$$

to estimate the entropy. Note that we use not $L_{n}(j)$ but $L_{j}(j)$ because we consider a finite string $x_{0}, \ldots, x_{n}$.

\section{A Combinatorial property of longest common prefix array}

In this section we give a new combinatorial property of the longest common prefix array. First we give definitions. Let $S=S[0] S[1] \ldots S[n]$ be a string. The suffix array [5] of $S$ is an integer array $I[0 . . n]$ such that $I[i]=j$ if $S_{j}=S[j] S[j+1] \ldots S[n]$ is lexicographically $i$-th suffix among all suffixes of $S$. The longest common prefix array is an array storing $l c p(I[i-1], I[i])+1$ for $i=0, \ldots, n$.

\subsection{Main result}

The following is the main theorem of this paper, which connects a combinatorial value defined on the suffix array with an information theoretic value appeared in the LZ77 compression algorithm.

\section{Theorem 2}

$$
\sum_{i=1}^{n}\{\operatorname{lcp}(I[i-1], I[i])+1\}=\sum_{j=0}^{n-1} L_{j}(j)
$$

To prove this theorem, we first show that the summation of $l c p$ 's in the lemma does not change after rearrangement of suffixes in the suffix array according to a particular order, then show that the equation holds in the rearranged array $I^{\prime}$.

We define an order $<_{L Z}$ for the rearrangement. The order of suffixes of a string for the rearrangement is defined as follows.

Definition 3 A suffix $S_{s}$ is smaller than a suffix $S_{t}$ in order of $<_{L Z}$ if the following condition holds. Let $l=$ $\operatorname{lcp}\left(S_{s}, S_{t}\right)$ and

$$
j_{s}=\underset{0 \leq j \leq n-1}{\operatorname{argmin}}\left\{j \mid l c p\left(S_{j}, S_{s}\right) \geq l+1\right\}
$$

$$
j_{t}=\underset{0 \leq j \leq n-1}{\operatorname{argmin}}\left\{j \mid l c p\left(S_{j}, S_{t}\right) \geq l+1\right\},
$$

then

$$
j_{s}<j_{t}
$$

Note that the string has a unique terminator $\$$ and therefore any suffix is not contained in other suffixes. Thus $l<\min \left\{\left|S_{s}\right|,\left|S_{t}\right|\right\}$ and the sets in the definition of $j_{s}$ and $j_{t}$ have at least one element, $s$ and $t$, respectively. Therefore $j_{s}$ and $j_{t}$ are well-defined.

To sort suffixes according to the order $<_{L Z}$, we first divide the suffixes according to their first characters into several groups and arrange the groups. The order of the groups is defined by minimum values of indices of the suffixes in the groups. These values correspond to $j_{s}$ and $j_{t}$ in the definition of $<_{L Z}$. We assume that suffixes $S_{a}$ and $S_{b}$ are in the same group $G_{1}$ and suffixes $S_{a}$ and $S_{c}$ are in different groups $G_{1}$ and $G_{2}$. Then $\operatorname{lcp}\left(S_{a}, S_{b}\right) \geq 1$ and $\operatorname{lcp}\left(S_{a}, S_{c}\right)=0$. Indices $j_{a}$ and $j_{c}$ in the definition of the order of $S_{a}$ and $S_{c}$ are equal for all suffixes $S_{a}$ in $G_{1}$ and $S_{c}$ in $G_{2}$. The indices $j_{a}$ and $j_{c}$ become the smallest indices in $G_{1}$ and $G_{2}$, respectively. This justifies the definition of order of groups.

Next we subdivide the suffixes in the groups according to their second characters. The order of the groups is also defined by minimum indices in the groups. We continue dividing suffixes until all suffixes belong to different groups.

Fig. 1 and Fig. 2 show an example of $I$ and $I^{\prime}$ for the string 'to be or not to be $\$$.' The longest-match suffix of a suffix $S_{s}=S_{4}$ is $S_{t}=S_{1}$. In Fig. 2, $t=I^{\prime}$ [3] and $s=I^{\prime}[5]$ and $L_{s}(s)=2$ appears in $l c p\left(I^{\prime}[5-1], I^{\prime}[5]\right)+$ 1. We see that in Fig. 2, $\operatorname{lcp}(I[i-1], I[i])+1=L_{s}(s)$ $\left(s=I^{\prime}[i]\right)$ for all $i$.

Proposition 1 The order $<_{L Z}$ is transitive, that is, if $S_{a}<_{L Z} S_{b}$ and $S_{b}<_{L Z} S_{c}$, then $S_{a}<_{L Z} S_{c}$ for all suffixes $S_{a}, S_{b}$, and $S_{c}$.

Proof: We assume that

$$
\begin{aligned}
l_{1} & =\operatorname{lcp}\left(S_{a}, S_{b}\right) \\
j_{a} & =\underset{0 \leq j \leq n-1}{\operatorname{argmin}}\left\{j \mid l c p\left(S_{j}, S_{a}\right) \geq l_{1}+1\right\} \\
j_{b 1}= & \underset{0 \leq j \leq n-1}{\operatorname{argmin}}\left\{j \mid l c p\left(S_{j}, S_{b}\right) \geq l_{1}+1\right\} \\
l_{2}= & \operatorname{lcp}\left(S_{b}, S_{c}\right) \\
j_{b 2}= & \underset{0 \leq j \leq n-1}{\operatorname{argmin}}\left\{j \mid l c p\left(S_{j}, S_{b}\right) \geq l_{2}+1\right\} \\
j_{c}= & \underset{0 \leq j \leq n-1}{\operatorname{argmin}}\left\{j \mid l c p\left(S_{j}, S_{c}\right) \geq l_{2}+1\right\} \\
l_{3}= & \operatorname{lcp}\left(S_{a}, S_{c}\right) \\
j_{a}^{\prime}= & \underset{0 \leq j \leq n-1}{\operatorname{argmin}}\left\{j \mid l c p\left(S_{j}, S_{a}\right) \geq l_{3}+1\right\}
\end{aligned}
$$




\begin{tabular}{|c|c|c|c|c|c|c|c|c|c|c|c|c|c|c|}
\hline \multirow{7}{*}{$s=I[i]$} & 0 & 1 & 2 & 3 & 4 & 5 & 6 & 7 & 8 & 9 & 10 & 11 & 12 & 13 \\
\hline & 13 & 11 & 2 & 12 & 3 & 6 & 10 & 1 & 4 & 7 & 5 & 9 & 0 & 8 \\
\hline & $\$$ & $\mathrm{~b}$ & $\mathrm{~b}$ & $\mathrm{e}$ & $\mathrm{e}$ & $\mathrm{n}$ & $\mathrm{o}$ & $\mathrm{o}$ & $\mathrm{o}$ & $\mathrm{O}$ & $\mathrm{r}$ & $\mathrm{t}$ & $\mathrm{t}$ & $\mathrm{t}$ \\
\hline & & $\mathrm{e}$ & $\mathrm{e}$ & $\$$ & $\mathrm{o}$ & & $\mathrm{b}$ & $\mathrm{b}$ & $\mathrm{r}$ & $\mathrm{t}$ & & $\mathrm{O}$ & $\mathrm{O}$ & $\mathrm{t}$ \\
\hline & & $\$$ & $\mathrm{o}$ & & $\mathrm{r}$ & & $\mathrm{e}$ & $\mathrm{e}$ & & & & $\mathrm{b}$ & $\mathrm{b}$ & \\
\hline & & & & & & & $\$$ & $\mathrm{o}$ & & & & $\mathrm{e}$ & $\mathrm{e}$ & \\
\hline & & & & & & & & & & & & $\$$ & $\mathrm{O}$ & \\
\hline $\operatorname{lcp}(I[i-1], I[i])+1$ & 1 & 1 & 3 & 1 & 2 & 1 & 1 & 4 & 2 & 2 & 1 & 1 & 5 & 2 \\
\hline$L_{s}(s)$ & 1 & 3 & 1 & 2 & 1 & 1 & 4 & 1 & 2 & 2 & 1 & 5 & 1 & 2 \\
\hline$B_{s}$ & 1 & 3 & 3 & 2 & 2 & 1 & 4 & 4 & 2 & 2 & 1 & 5 & 5 & 2 \\
\hline
\end{tabular}

Fig. 1 Alphabetic order.

\begin{tabular}{|c|c|c|c|c|c|c|c|c|c|c|c|c|c|c|}
\hline \multirow{7}{*}{$s=I^{\prime}[i]$} & 0 & 1 & 2 & 3 & 4 & 5 & 6 & 7 & 8 & 9 & 10 & 11 & 12 & 13 \\
\hline & 0 & 9 & 8 & 1 & 10 & 4 & 7 & 2 & 11 & 3 & 12 & 5 & 6 & 13 \\
\hline & $\mathrm{t}$ & $\mathrm{t}$ & $\mathrm{t}$ & $\mathrm{o}$ & $\mathrm{o}$ & $\mathrm{o}$ & $\mathrm{o}$ & $\mathrm{b}$ & $\mathrm{b}$ & $\mathrm{e}$ & $\mathrm{e}$ & $\mathrm{r}$ & $\mathrm{n}$ & $\$$ \\
\hline & $\mathrm{O}$ & $\mathrm{O}$ & $\mathrm{t}$ & $\mathrm{b}$ & $\mathrm{b}$ & $\mathrm{r}$ & $\mathrm{t}$ & $\mathrm{e}$ & $\mathrm{e}$ & $\mathrm{o}$ & $\$$ & & & \\
\hline & $\mathrm{b}$ & $\mathrm{b}$ & & $\mathrm{e}$ & $\mathrm{e}$ & & & $\mathrm{o}$ & $\$$ & & & & & \\
\hline & $\mathrm{e}$ & $\mathrm{e}$ & & $\mathrm{O}$ & $\$$ & & & & & & & & & \\
\hline & $\mathrm{o}$ & $\$$ & & & & & & & & & & & & \\
\hline $\operatorname{lcp}\left(I^{\prime}[i-1], I^{\prime}[i]\right)+1$ & 1 & 5 & 2 & 1 & 4 & 2 & 2 & 1 & 3 & 1 & 2 & 1 & 1 & 1 \\
\hline$L_{s}(s)$ & 1 & 5 & 2 & 1 & 4 & 2 & 2 & 1 & 3 & 1 & 2 & 1 & 1 & 1 \\
\hline$B_{s}$ & 5 & 5 & 2 & 4 & 4 & 2 & 2 & 3 & 3 & 2 & 2 & 1 & 1 & 1 \\
\hline
\end{tabular}

Fig. $2<_{L Z}$ order.

$$
\begin{aligned}
j_{c}^{\prime} & =\underset{0 \leq j \leq n-1}{\operatorname{argmin}}\left\{j \mid l_{c p}\left(S_{j}, S_{c}\right) \geq l_{3}+1\right\} \\
j_{a} & <j_{b 1} \\
j_{b 2} & <j_{c} .
\end{aligned}
$$

We show that $j_{a}^{\prime}<j_{c}^{\prime}$. There are three cases.

Case $l_{1}<l_{2}: \operatorname{lcp}\left(S_{b}, S_{c}\right)=l_{2} \leq l_{1}+1$ and $l_{3}=l_{1}$. Therefore $j_{a}=j_{a}^{\prime}$, and $j_{b 1}$ and $j_{c}^{\prime}$ become as follows:

$$
\begin{aligned}
& j_{b 1}=\underset{j}{\operatorname{argmin}}\left\{j \mid l c p\left(S_{j}, S_{b}\right) \geq l_{1}+1,0 \leq j \leq n-1\right\} \\
& j_{c}^{\prime}=\underset{j}{\operatorname{argmin}}\left\{j \mid l c p\left(S_{j}, S_{c}\right) \geq l_{1}+1,0 \leq j \leq n-1\right\} .
\end{aligned}
$$

Because $\operatorname{lcp}\left(S_{b}, S_{c}\right) \leq l_{1}+1$, definitions of $j_{b 1}$ and $j_{c}^{\prime}$ coincide and therefore $j_{b 1}=j_{c}^{\prime}$. These show that $j_{a}^{\prime}=j_{a}<j_{b 1}=j_{c}^{\prime}$.

Case $l_{1}>l_{2}$ : we can show that $j_{a}^{\prime}=j_{b 2}<j_{c}=j_{c}^{\prime}$ in a similar way.

Case $l_{1}=l_{2}:$ From the definition $j_{b 1}=j_{b 2}$ and $l 3 \geq$ l1. If $l_{3}=l_{1}=l_{2}, j_{a}^{\prime}=j_{a}<j_{b 1}=j_{b 2}<j_{c}=j_{c}^{\prime}$. If $l_{3}>l_{1}=l_{2}, \operatorname{lc} p\left(S_{a}, S_{c}\right) \geq l_{1}+1$ and definitions of $j_{a}$ and $j_{c}$ become as follows:

$$
\begin{aligned}
& j_{a}=\underset{j}{\operatorname{argmin}}\left\{j \mid l c p\left(S_{j}, S_{a}\right) \geq l_{1}+1,0 \leq j \leq n-1\right\} \\
& j_{c}=\underset{j}{\operatorname{argmin}}\left\{j \mid l c p\left(S_{j}, S_{c}\right) \geq l_{1}+1,0 \leq j \leq n-1\right\} .
\end{aligned}
$$

These definitions coincide and therefore $j_{a}=j_{c}$. Then $j_{b 2}<j_{c}=j_{a}<j_{b 1}$, which contradicts $j_{b 1}=j_{b 2}$. It means that the case $l_{3}>l_{1}=l_{2}$ does not occur.
In all cases, the inequality $j_{a}^{\prime}<j_{c}^{\prime}$ holds.

After this rearrangement, the summation of $l c p$ 's between adjacent suffixes in $I^{\prime}$ is equal to that in the original suffix array $I$.

\section{Lemma 1}

$$
\sum_{i=1}^{n} \operatorname{lcp}(I[i-1], I[i])=\sum_{i=1}^{n} \operatorname{lcp}\left(I^{\prime}[i-1], I^{\prime}[i]\right)
$$

Proof: We show that $l c p$ 's of adjacent suffixes in $I^{\prime}$ are a permutation of that in $I$. After dividing suffixes into groups according to the first characters, $l c p$ 's between different groups are zero and they do not depend on sorting order. Therefore the number of zeroes in $l c p$ 's does not depend on sorting order. Then suffixes in each group are subdivided according to the second characters. After the subdivision $l c p$ between two subgroups in a group is one regardless of sorting order. Therefore the number of ones in $l c p$ 's does not change. We can prove that the number of $l$ in $l c p$ 's between adjacent suffixes in $I^{\prime}$ is always equal to that in $I$ in a similar way.

Now we can show a relationship between $l c p$ and $L_{s}(s)$. Note that we use $L_{s}(s)$ instead of $L_{n}(s)$.

Lemma 2 Concerning a suffix $S_{s}\left(s=I^{\prime}[i]\right)$, a suffix $S_{t}$ which corresponds to $L_{s}(s)$ is located in smaller position than $S_{s}$ in the array $I^{\prime}$, and

$$
\operatorname{lcp}\left(I^{\prime}[i-1], I^{\prime}[i]\right)+1=L_{s}(s) .
$$


Note that the suffix $S_{t}$ may not be adjacent to $S_{s}$.

Proof: From the definition of the order $<_{L Z}$, one of suffixes which are the longest-match suffixes of $S_{s}$ is located in $I^{\prime}[i-1]$ or $I^{\prime}[i+1]$. Suffixes which are larger than $S_{s}$ in order of $<_{L Z}$, that is, suffixes $I^{\prime}[i+k](k=1,2, \ldots)$ do not correspond to $L_{s}(s)$ even if $l c p\left(I^{\prime}[i], I^{\prime}[i+1]\right)>l c p\left(I^{\prime}[i], I^{\prime}[i-1]\right)$. The reason is as follows. We assume that a suffix $S_{u}$ is in $I^{\prime}[i+k](k=1,2, \ldots)$. Let $l c p\left(S_{s}, S_{u}\right)=l$ and

$$
\begin{aligned}
& j_{s}=\underset{0 \leq j \leq n-1}{\operatorname{argmin}}\left\{j \mid l c p\left(S_{j}, S_{s}\right) \geq l_{1}+1\right\} \\
& j_{u}=\underset{0 \leq j \leq n-1}{\operatorname{argmin}}\left\{j \mid l c p\left(S_{j}, S_{u}\right) \geq l_{1}+1\right\} .
\end{aligned}
$$

Since $S_{s}<_{L Z} S_{u}, j_{s}<j_{u}$. It means that the suffix $S_{u}$ is not a suffix which corresponds to $L_{s}(s)$.

Therefore suffixes $I^{\prime}[i-k](k=1,2, \ldots)$ are candidates for the longest-match suffix of $S_{s}$. Some of them are excluded because their positions are larger than $s$. However, there exists at least one suffix $S_{t}$ for each suffix $S_{s}$ except $S_{0}$ such that $t<s$ and $\operatorname{lcp}\left(S_{t}, S_{s}\right)=$ $\operatorname{lcp}\left(I^{\prime}[i-1], I^{\prime}[i]\right)$. Since $L_{s}(s)=\operatorname{lcp}\left(S_{t}, I^{\prime}[i]\right)+1$, $\operatorname{lcp}\left(I^{\prime}[i-1], I^{\prime}[i]\right)+1=L_{s}(s)$.

The proof of Theorem 2 is obvious from Lemma 1 and Lemma 2.

\subsection{Linear time algorithm}

The value $\sum_{j=0}^{n-1} L_{j}(j)$ is computed in linear time as follows. First we construct the suffix array $I$ in linear time using a practical linear time algorithm [6]. Then the $l c p$ array can be also computed in linear time [7]. It is obvious to obtain the desired value from the $l c p$ array in linear time.

\subsection{Relation with distinguishing prefixes}

We also show the relation between the summation of $B_{j}$ and the summation of $L_{j}(j) . B_{j}$ can be computed by

$$
\begin{array}{r}
B_{j}=\max \{\operatorname{lcp}(I[i-1], I[i])+1, \\
\operatorname{lcp}(I[i], I[i+1])+1\} .
\end{array}
$$

Therefore the entropy estimation with this scheme is also done in linear time.

We express an upper-bound of the summation of $B_{j}$ 's for all suffixes by the summation of $l c p$ 's between adjacent suffixes in the suffix array.

\section{Lemma 3}

$$
\sum_{j=0}^{n-1}\left(B_{j}-1\right) \leq 2 \sum_{i=1}^{n} l c p(I[i-1], I[i])
$$

Proof: By using an inequality

$$
\max \{a, b\} \leq a+b \quad(a, b \geq 0),
$$

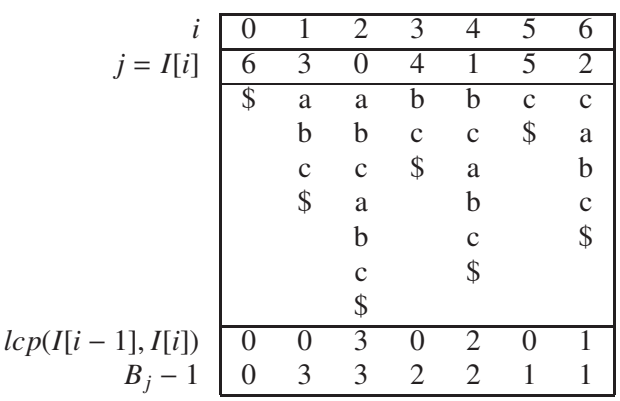

Fig. 3 An example of a string.

$$
\begin{aligned}
& \sum_{j=0}^{n-1}\left(B_{j}-1\right) \\
& \quad=\sum_{i=1}^{n} \max \{\operatorname{lcp}(I[i-1], I[i]), \operatorname{lcp}(I[i], I[i+1])\} \\
& \quad \leq \sum_{i=1}^{n}\{l c p(I[i-1], I[i])+\operatorname{lcp}(I[i], I[i+1])\} \\
& \quad=2 \sum_{i=1}^{n} \operatorname{lcp}(I[i-1], I[i])
\end{aligned}
$$

Note that $l c p(I[0], I[1])=l c p(I[n], I[n+1])=0$.

The equality holds when the string $X$ forms concatenation of the same two strings in which all characters differ, for example 'abc...zabc...z\$.' Fig. 3 shows an example of a suffix array for such a string. for a string of length $2 n$. In such cases,

$$
\begin{aligned}
& \sum_{i=1}^{2 n} \operatorname{lcp}(I[i-1], I[i])=\frac{n(n+1)}{2} \\
& \sum_{j=0}^{2 n-1} B_{j}=n(n+1)
\end{aligned}
$$

This means that estimated values of entropy by $B_{j}$ is sometimes far from that by $L_{j}(j)$.

\section{References}

[1] H. Yokoo, "Context tables: a tool for describing text compression algorithms," In Proc. of IEEE Data Compression Conference, pp.299-308, 1998.

[2] H. Yokoo, "A Dynamic data structure for reverse lexicographically sorted prefixes," In M. Crochemore and M. Paterson, editors, Proc. of the 10th Annual Symposium on Combinatorial Pattern Matching (CPM'99), LNCS 1645, pp.150-162, 1999.

[3] J. Ziv and A. Lempel, "A Universal algorithm for sequential data compression," IEEE Trans. Inform. Theory, vol.IT-23, no.3, pp.337-343, 1977. 
[4] A. D. Wyner and J. Ziv, "Some Asymptotic Properties of the Entropy of a Stationary Ergodic Data Source with Applications to Data Compression," IEEE Trans. Inform. Theory, vol.IT-35, no.6, pp.1250-1258, 1989.

[5] U. Manber and G. Myers, "Suffix arrays: A new method for on-line string searches," SIAM Journal on Computing, vol.22, no.5, pp.935-948, 1993.

[6] G. Nong, S. Zhang, and W. H. Chan, "Linear suffix array construction by almost pure induced-sorting," In $D C C$, pp.193-202, 2009.

[7] S. Gog and E. Ohlebusch, "Fast and lightweight lcp-array construction algorithms," In Proc. ALENEX, pp.25-34, 2011.

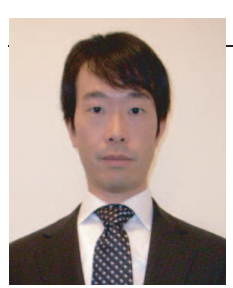

Kunihiko SADAKANE

Kunihiko SADAKANE received B.S., M.S., and Ph.D. degrees from Department of Information Science, University of Tokyo in 1995, 1997 and 2000 , respectively. He was a research associate at Graduate School of Information Sciences, Tohoku University from 2000 to 2003, and an associate professor at Faculty of Information Science and Electrical Engineering, Kyushu University from 2003 to 2009. Since 2009, he has been an associate professor at National Institute of Informatics. His research interest includes information retrieval, data structures, and data compression. He is a member of IPSJ and IEICE. 